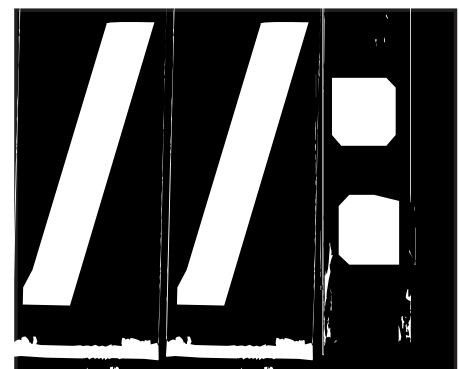

aduca"ción y cơmunicación 5: 67-81 Nov. 2012

\section{EDUCACIÓN Y COMUNICACIÓN: LA RADIO COMO CONSTRUCTORA DE CIUDADANÍA Y POTENCIADORA DE LAS RELACIONES ESCUELA-ENTORNO.}

\section{Education and Communication: Radio as a Constructing Gitizenship and Fomenting School-Environment Relations}

Oscar Arcera López

Máster en Formación del Profesorado de Secundaria. Escuela Solidaria Iqbal Masih, Torremocha de Jarama, MADRID (España) E.mail: oscararceralopez@yahoo.es

Resumen:

La radio en la escuela o la radio educativa, no es evidentemente un tema nuevo. Sólo remontándonos al intento de impulso por parte de la UNESCO para que se integrara la educación en medios de comunicación dentro del sistema escolar, llegamos a los años 80 con la Declaración de Grünewald. Y mucho antes ya germinaban experiencias de radio educativa y/o comunitaria por diversos puntos de la geografía mundial. Pero lo que sigue siendo nuevo, actual, e incluso todo un reto para los que nos planteamos la tarea de la educación y/o de la comunicación es la integración de ambos en un solo proceso (educomunicación).

El presente artículo trata de mostrar la improrrogable urgencia de esa relación dialéctica entre dos instituciones que tienen tendencia a ignorarse mutuamente, la Escuela y los Medios de Comunicación Social. Y lo hace a través de una reflexión pedagógica iluminada por algunas experiencias históricas y una praxis educativa en el seno de una radio juvenil protagonizada por jóvenes, radio ESIM.

Palabras clave: Educación, comunicación, jóvenes, protagonismo, creatividad, compromiso, conciencia crítica, responsabilidad.

\section{Abstract:}

School radio as an educative medium is not a recent phenomenon. A cursory glance to the past brings us to the UNSECO initiative to integrate education within the media, in the spirit of the Grünewald Declaration. These kinds of initiatives have been previously present throughout the world. But what can still be considered an innovation and a challenge nowadays is to integrate both into a single process (educommunication).

This article sets out to show the absolute urgency that exists to bring together schooling practices and the social communication media. This can be brought about through a pedagogical reflection on previous experiences in the field and through an educative praxis within a juvenile radio, where the young are protagonist. This is radio ESIM. Key words: Education, communication, youth, creativity, critical, commitment, consciousness, responsibility.

Recibido: 15-09-2012 / Revisado: 20-09-2012 / Aceptado: 30-09-2012 / Publicado: 01-11-2012

https://dx.doi.org/10.25267/Hachetetepe.2012.v2.i5.7 


\section{Introducción}

T os Medios de Comunicación, -radio, televisión, Uprensa, Webs, blogs y foros, redes sociales, y los últimos avances en comunicación a través de la telefonía móvil-, son agentes excepcionalmente eficaces de educación y/o de contraeducación. Nos guste o no, la realidad, y así lo señalan las estadísticas, es que los jóvenes pasan más tiempo frente al ordenador, la televisión, escuchando música, que las que pasan dentro de la educación formal. El poder de la "educación" informal es hoy mayor que nunca, ya que a la omnipresencia de los medios de comunicación tradicionales, se han sumado en los últimos años la creciente influencia de las redes sociales, las tecnologías 2.0 y los excepcionales avances en la nanotecnología que, cómo no, han revolucionado el campo de las comunicaciones en general. $Y$ en ese poder educativo de lo externo a la escuela, o en la terminología de Trilla Bernet (2003), la educación fuera de la escuela, juega un papel fundamental los Mass-Media.

Por ello, en el aula, los Medios deben ser mucho más que meros "recursos" auxiliares que pudieran ponerse a disposición del profesorado para ampliar cuantitativamente el número de los destinatarios de su actividad docente o para mejorar cualitativamente los resultados de nuestro proceso de enseñanza. Sin que quepa minusvalorar estas dimensiones, los medios de comunicación suponen, en relación con el sistema educativo, una palanca excepcional para la ampliación del horizonte cuantitativo de los destinatarios de la actividad docente: su acción puede alcanzar a la población, en edad escolar o no, mucho más allá de los muros de la escuela: a las familias, al barrio, a los propios escolares fuera del horario escolar...
Los Medios significan todo eso, pero por ello, mucho más que eso. Sólo ellos pueden convertir en expectativa razonable, en proyecto sensato, la "utopía" de la Educación Permanente, hasta ahora mero axioma ligado a razonamientos teóricos. Una permanente y universal oferta de servicios educativos es ya posible desde las potencialidades de los Medios de Comunicación (páginas Web, blogs, el periódico de la escuela, la radio del IES), más allá de las inesquivables dificultades que para ese objetivo representan los recursos humanos y económicos de la educación tradicional. Y también son capaces, por su propia naturaleza, de convertir en expectativa razonable la "utopía" de la Escuela abierta a la sociedad y al entorno que la rodea. A este respecto resultan suficientemente clarificadoras las siguientes palabras de Cervera (1977):

«Con visión pedagógica, todo educador considerará que el fenómeno de los medios de comunicación de masas y la iniciación a las técnicas de comunicación no es ajeno al campo de la educación; pues si es verdad que hace tiempo que la escuela perdió el monopolio de la educación, hoy recobra un estilo actualizado de escuela abierta al medio socio-cultural, sin muros, que la hace participativa y atractiva»

Con excesiva frecuencia, pretendemos establecer una alternativa entre la educación tradicional y la propiciada por las nuevas tecnologías, como si hubiera que elegir entre la enseñanza presencial tradicional -y sus valores- y la enseñanza posibilitada por los Medios y/o las TIC -y los suyos-. No existe alternativa, pero sí relación dialéctica entre una y otra. Parecen oponerse si se considera sólo una y otra de sus dimensiones, pero realmente se complementan y necesitan mutuamente 
para su máxima eficacia. Las investigaciones lo han puesto de manifiesto. No es sólo que la educación tradicional se vea beneficiada por la incorporación de los Medios a su elenco de recursos didácticos; es que el impacto de los Medios profundiza su eficacia desde el momento en que cuenta con el apoyo del educador que ayuda al alumno a tener una posición crítica en su lectura de la "realidad" ofrecida por los medios. El reto no es, por tanto, la sustitución de un tipo de educación por otro, ni siquiera la complementación de uno con el otro. El reto es la integración de ambos en un solo proceso, integración que reclama nuestro trabajo decidido para que, los mensajes de nuestro sistema escolar junto a los que proporcionan los Medios, unos y otros con frecuencia no coincidentes y no pocas veces contradictorios, adquieran la coherencia necesaria para que nuestros alumnos adquieran verdaderos aprendizajes significativos. Es cierto que el sistema educativo está ahí con toda evidencia, formando y educando a nuestras nuevas generaciones de jóvenes. Pero no es menor la evidencia del hecho mediático, que cada día -en el conjunto del año en mayor número de horas que la duración de la jornada escolar- proyecta sus mensajes sobre la población escolar y adulta, con una capacidad de persuasión y de motivación que quisiera para sí la didáctica tradicional. Por ello resulta improrrogablemente urgente la conexión entre Educación y Medios de Comunicación.

«La comunicación se manifiesta como un fenómeno que genera un "medio educativo", parece arrebatar a la escuela su monopolio educativo y la induce a utilizar en provecho propio las formas modernas de la comunicación» (Dieuzeide, 1984)

\section{La omnipresencia de los medios de comunicación y nuestra responsabilidad como educadores}

Hace más de 30 años, Dieuzeide (1984) ya anunciaba la aparición de un hombre nuevo cuyos hábitos lógicos, universo afectivo, actitudes críticas, mundo imaginario, destrezas técnicas y comportamientos podrían ser modelados día a día por ese medio ambiente. Ese hombre nuevo está ya en nuestras aulas. Los adultos, estamos acostumbrados a establecer distinciones entre las funciones (televisión, Internet, radio, teléfono móvil) y no llegamos a percibir bien las conexiones que ya han realizado los progresos de la electrónica en la vida cotidiana y que hace unos años eran incompatibles; hoy Internet nos llega, no sólo ya a través de los cada vez más perfeccionados dispositivos informáticos clásicos, sino a través de nuestro teléfono y de nuestro televisor, desde el cual ya no sólo vemos la programación, sino que consultamos nuestro correo y hablamos por teléfono con cualquier lugar del mundo a precio de llamada local a través de Skype. Nuestros jóvenes de hoy se despiertan con el sonido de un WhatsApp, escuchan la radio en su teléfono mientras van de camino al IES, consultan los últimos mensajes y vídeos de YouTube mientras "acuden" a nuestras clases, y continúan el resto del día, ya fuera del centro escolar, enganchados a su red social de "amigos" -virtuales en gran número-.

Ya no es en la escuela -que, sin embargo, debería ser el medio de la reflexión y de la transferencia de conocimientos- donde se familiariza con los conceptos de base que subyacen en el universo tecnológico de hoy. En realidad la omnipresencia de la comunicación no es sino el signo de la creación de un nuevo medioambiente. Lo que en la Teoría 
ecológica de Bronfenbrenner (1987) sería un ecosistema "formativo" con claras repercusiones sobre todos los demás (escuela, familia, barrio,...) y mutuas interrelaciones entre todos ellos.

Por otro lado, los medios de comunicación parece que condujesen a una cierta estandarización intelectual. Sin embargo, nada permite pensar que ese movimiento hacia la estandarización que caracteriza hoy a la mayor parte de las industrias de comunicación sea inexorable. Aquí es donde radica nuestra responsabilidad como educadores. Aquí es donde radica la responsabilidad de la Escuela, si esta quiere, en cuanto a que debe dar respuesta a las necesidades de la sociedad en la que se encuentra inmersa. En este sentido Trilla Bernet (2003) afirma:

«La escuela ha de intentar dar respuesta a las necesidades educativas de nuestra sociedad. Ahora bien, hay que entender que esta institución por ella misma ni podría hacerlo ni es la única que tiene tal responsabilidad. Es por ello que conviene situar la acción escolar en el marco más amplio del conjunto de agencias que configuran el universo de la educación»

Pero la realidad, en la mayor parte de los casos, ha sido que la escuela, como "sociedad de comunicación", ha intentado de forma selectiva y voluntarista someter la mayor parte de las formas modernas de comunicación a sus propios objetivos: ya sea solicitando la ayuda de los sistemas de comunicación en su estado actual para difundir una información de valor educativo entre el público habitual de dichos sistemas, ya sea utilizando esos mismos sistemas de comunicación para introducir componentes nuevos en actividades de educación formal (radio, televisión, prensa, películas escolares). Este segundo camino de integración de los medios de comunicación al currículum escolar constituye un paso importante, pero no suficiente. Pues, por encima del instrumento en sí, pongamos como caso la radio escolar, está nuestra responsabilidad de enseñar a todos el uso ponderado de la comunicación. Esta necesidad se hace tanto más acuciante cuanto que la familia ha demostrado su falta de preparación para enfrentarse con la irrupción de la comunicación.

\section{0 años de la Declaración de Grunëwald. Una breve revisión de la radio educativa y/o social a la luz de sus postulados.}

Este año 2012 se cumplen 30 años de la Declaración de Grünewald. Alemania, en la que, por primera vez, en el marco de un Simposio internacional, la UNESCO se pronuncio de forma inequívoca sobre la necesidad de la inserción de la Educación en Medios en el sistema escolar. El texto, que fue firmado por 19 países, planteaba, ya entonces, que vivimos en un mundo en que los medios de comunicación están omnipresentes: un número de personas cada vez mayor consagra buena parte de su tiempo a ver la televisión, a leer diarios y revistas, a escuchar grabaciones sonoras o la radio [hoy tendríamos que añadir los nuevos medios aparecidos con los recientes avances tecnológicos]. Consideraba que en lugar de condenar o aprobar el indiscutible poder de los medios de comunicación, es necesario aceptar como un hecho establecido su considerable impacto y su propagación a través del mundo y reconocer al mismo tiempo que constituyen un elemento importante de la cultura en el mundo contemporáneo. La declaración de Grünewald no era la primera llamada de la UNESCO a la necesidad de incorporar a los medios de comunicación a la 
educación, pero significó una mayor concreción de sus propuestas. Ante la evidencia de que la progresiva importancia de los medios en la vida de los ciudadanos no tenía correlación con su presencia en los sistemas educativos, se hicieron varias recomendaciones para que fuesen implementadas por los gobiernos:

1. Organizar y apoyar programas integrados de educación relativa a los medios de comunicación desde el nivel preescolar hasta el universitario y la educación de adultos, con vistas a desarrollar los conocimientos, técnicas y actitudes que permitan favorecer la creación de una conciencia crítica $y$, por consiguiente, de una mayor competencia entre usuarios de los medios de comunicación electrónicos e impresos. Lo ideal sería que esos programas abarcaran desde el análisis de contenido de los medios de comunicación hasta la utilización de los instrumentos de expresión creadora, sin dejar de lado la utilización de los canales de comunicación disponibles basada en una participación activa.

2. Desarrollar cursos de formación para los educadores $\mathrm{y}$ diferentes tipos de animadores y mediadores, encaminados tanto a mejorar el conocimiento $\mathrm{y}$ comprensión de los medios de comunicación como a que se familiaricen con métodos de enseñanza apropiados que tengan en cuenta el conocimiento de los medios de comunicación, a menudo considerable pero aún fragmentario, que poseen ya la mayoría de los estudiantes.

3. Estimular las actividades de investigación y desarrollo en educación relativa a los medios de comunicación.
4. Apoyar y reforzar las medidas adoptadas o previstas por la UNESCO con miras a fomentar la cooperación internacional dentro de la esfera de la educación relativa a los medios de comunicación.

Posteriormente en los seminarios organizados por la UNESCO en Sevilla (febrero 2002), al que siguieron el encuentro en Tesalónica (marzo 2003) y en El Cairo (diciembre 2003) se han adoptado varias decisiones operativas para trabajar en ese ámbito:

- La Educación en medios trata sobre la enseñanza y el aprendizaje CON y SOBRE los medios, más que A TRAVÉS de los medios.

- Implica tanto el análisis crítico como la producción creativa.

- Puede tener lugar en la educación formal y no formal.

- Debe promover el sentido comunitario y de responsabilidad social, así como el crecimiento y la realización personal.

Un rápido y somero análisis de las diferentes experiencias de radio educativa y/o social que se pusieron en marcha al tiempo, o coincidiendo con el impulso de la UNESCO (algunas de ellas aún en marcha), nos permite afirmar que:

En los países empobrecidos o en vías de desarrollo, donde el medio cultural era más pobre, se ha producido una mayor implantación de la radio educativa $\mathrm{y}$ comunitaria. A pesar de esta característica común, se pueden distinguir algunas diferencias entre diferentes continentes: 
- En África la utilización del medio radiofónico ha sido mayoritariamente para ampliar el nivel de alfabetización, paliando así las deficiencias en infraestructuras educativas, la escasez de maestros y educadores y la realidad de una población dispersa, fundamentalmente en los núcleos rurales. En cuanto a su incorporación real en la enseñanza formal, a pesar de algunos pasos en esa dirección como en el caso de Ghana, ha faltado, como en otros países, una adaptación curricular de su sistema educativo. Sólo una reforma en este sentido puede lograr que las nuevas tecnologías de la información y la comunicación no se pongan al servicio de antiguas prácticas y metodologías educativas.

- En Iberoamérica, un continente con una larga tradición de radio educativa y comunitaria, es donde más se ha extendido el uso de la radio desde la perspectiva que se plantea en este artículo. A nivel educativo, son muchas las escuelas, asociaciones, que cuentan con su pequeña emisora de radio. También la radio comunitaria (Radio Sutatenza, Radio Favela) ha tenido una gran importancia para el desarrollo social de ese país, además de una importante repercusión internacional. No podemos olvidar en este punto que muchas otras experiencias diseminadas a lo largo y ancho del mundo (véase Radio ECCA) son fruto de la exportación del modelo iberoamericano. En estos países la lucha fundamental, en cuanto a las radios comunitarias, ha sido la de no sucumbir a los tentáculos del Estado y a las seducciones del Mercado. A nivel educativo su mayor dificultad ha sido la falta de libertad en un gran número de países con regímenes no democráticos.
- En Europa, al igual que en el caso particular de España, se ha trabajado fundamentalmente en la dirección de intentar hacer llegar la educación a la población físicamente dispersa, o que por motivos de horarios laborales no podía acceder a la misma. La radio sirvió más para un aumento cuantitativo de los destinatarios de la educación formal, que para una mejora cualitativa de la misma. Se llegó a introducir la radio dentro del currículum de la enseñanza formal (Bachillerato radiofónico), pero fue difícil mantener el volumen de trabajo que exigen proyectos de esta envergadura con una estructuración marcadamente disciplinar.

\section{En resumen...}

Han existido a lo largo del tiempo, y se siguen desarrollando algunas en la actualidad, muchas prácticas educativas destinadas a preparar un consumo apropiado de la comunicación. Unas se han dedicado fundamentalmente a enseñar a utilizar la información como un producto de consumo individual y otras a fomentar la utilización creativa de una comunicación concebida esencialmente como un proceso social. En esta perspectiva de un consumo más apropiado de los medios, se han desarrollado diferentes metodologías y programas cómo la utilización de periódicos en la escuela como textos de estudio, la iniciación a la comunicación audiovisual, la creación de emisoras escolares, tema que nos ocupa, la colaboración en las Webs escolares o el desarrollo de blogs de las asignaturas, como instrumentos que pueden ayudar a apreciar y a juzgar los mensajes, a descifrar las intenciones de los autores, a distinguir lo real de lo imaginario, a organizar y a seleccionar.

La comunicación ya no es el monopolio de los 
agentes. Todos deben iniciarse en la producción de mensajes audiovisuales, impresos o radiofónicos. Este enfoque "participativo", inaugurado antaño por el periódico escolar y la imprenta en la escuela, es el que ha llevado a un gran número de escuelas, de asociaciones educativas o de movimientos juveniles, a nuevos enfoques creativos, críticos y funcionales de los medios de comunicación, concretándose en el desarrollo de emisoras escolares o asociativas.

Sin embargo, esta relativa abundancia de iniciativas no ha cuajado todavía en estrategias pedagógicas coherentes y queda mucho por hacer aún a ese respecto. Algunos educadores, a lo largo de todo este complejo desarrollo, han creído conveniente que las tecnologías de la información y la comunicación y su empleo debían formar parte de nuevas disciplinas, aunque se corriera el riesgo de reforzar la compartimentación de las materias; para otros, el dominio de los medios debe aprenderse dentro de cada disciplina de enseñanza general, a riesgo de introducir a la fuerza en el ámbito escolar una cultura que quizás le resulte inaceptable. Lo que sí que parece claro es que, ni las políticas, ni los contenidos, ni los métodos de educación, ni la formación inicial o permanente de los profesores, ni la investigación educativa, pueden ignorar la problemática surgida del enfrentamiento entre la educación y la comunicación. Y aún menos que los otros, los países desheredados, pues su indigencia los expone sin la menor defensa a los efectos corrosivos de la explosión de la información que conmovió el fin del siglo XX y seguirá revolucionando la educomunicación en el siglo XXI.

\section{Queda mucho aún por hacer}

Sigue siendo hoy más necesario que nunca, si en verdad queremos que nuestros jóvenes de hoy puedan ser ciudadanos comprometidos en la sociedad del conocimiento de mañana, una correcta adecuación de nuestros procesos de enseñanza-aprendizaje, de forma que potenciando el uso de pedagogías que favorezcan la creación de una conciencia crítica, les permita no sólo un adecuado filtrado de las toneladas de información relevante e irrelevante que reciben cada día, sino la creación de sus propios medios como forma de protagonismo, responsabilidad y expresión creadora. Es necesaria una educación más crítica, capaz de denunciar al tiempo los peligros de un pseudosaber audiovisual y la ilusión del poder informático. Se trata de liberar al joven de la fascinación tecnológica y de volverlo más riguroso, más exigente, más consciente. $\mathrm{Y}$ es especialmente necesaria esta educación más crítica por dos motivos fundamentales.

En primer lugar porque como educadores tenemos esa responsabilidad. Nuestra tarea, atendiendo a la etimología de nuestra vocación profesional, es la de sacar de dentro de los educandos, lo mejor de cada uno, y la de guiar a cada uno de ellos por el camino de la Libertad y la Responsabilidad. Un camino a través del cual, educando y educador tendrán que ir tomando postura ante la realidad impuesta por la vida. Un camino a través del cual, educando y educador (y todos lo somos), a la sombra siempre de esas "fuerzas invisibles" que conforman y condicionan nuestro pensar y nuestro obrar, nos moveremos confusamente entre la objetividad y el subjetivismo, o entre la subjetividad y el objetivismo (Freire, 2000).

$\mathrm{Y}$ en segundo lugar e íntimamente relacionado con 
el párrafo anterior, la pretendida neutralidad ante la naturaleza política inherente a la tarea educativa, ha sido una de las causantes de la "crisis" de la institución escolar. (Cervera, 1977: 13). A este respecto Freire (1990) señala que el elemento político de la educación es independiente de la subjetividad del educador; es decir, es independiente de que el educador sea consciente de dicho factor, que jamás es neutral.

No obstante, no debemos caer en una visión derrotista a la luz de experiencias fracasadas o malogradas. Las posibilidades del aprendizaje con y sobre los medios de comunicación, en el ámbito de la escuela, están a nuestro alcance. Nunca las condiciones tecnológicas fueron tan favorables como ahora. Nunca la formación inicial del profesorado y sus posibilidades de ampliar esta formación de una forma continua y a lo largo de toda la vida, fueron tan favorables. Sólo son necesarios equipos de personas comprometidas con la entusiasmante tarea de educar. Educadores, padres, miembros de la amplia comunidad educativa que quieran comprometer su tiempo para hacer posible proyectos en los que los jóvenes sean protagonistas de su proceso educativo. Proyectos en los que el protagonismo, la participación, la creatividad, sean, además de fuente de aprendizaje, testimonio de una escuela abierta y comprometida con la sociedad en general, y con el entorno en el que se inserta en particular.

Al igual que Amar (2010), coincidimos con Área (1995) cuando afirma sobre los medios de comunicación que nadie duda de su "poderosa influencia sobre los ciudadanos y de su importante potencial pedagógico", lo que se necesita es utilizarlos como un arte (sinónimo de virtud, disposición y habilidad). No podemos caer en el derrotismo, en él no se puede hacer nada. No podemos caer en la tentación de revisar las experiencias históricas para quedarnos en los fallos, en las deficiencias y en las limitaciones. En cambio, podemos plantear líneas de actuación, nuevas propuestas, nuevas posibilidades, que a la luz de la experiencia, puedan aportar luz a una realidad innegable: la necesidad de una educación crítica y comprometida en la que creemos que, la radio y la escuela, como comunidad educativa, tienen mucho aún que aportar.

\section{Una realidad concreta: radio ESIM, una radio joven, hecha por jóvenes.}

ESIM son las siglas de la Escuela Solidaria Iqbal Masih, un proyecto educativo del Movimiento Cultural Cristiano que se sustenta en el protagonismo de sus tres pilares fundamentales: los jóvenes, los educadores y las familias. En el seno de este amplio proyecto educativo, véase Medina (2010), germina en el 2006 el proyecto de radio ESIM, en Torremocha de Jarama (Madrid). Comenzó como un mero taller de radio. Pretendía dar un paso más en el trabajo que ya veníamos haciendo cotidianamente sobre el análisis crítico de la información, a través de nuestro taller de prensa semanal. Y resultó un éxito, pues después de algunos talleres puntuales, fue demandado por los propios jóvenes de la Casa Escuela Iqbal Masih (12 a 18 años), para que pasara a formar parte de la programación semanal de actividades de nuestra humilde doble-escuela, en la más pura terminología milaniana.

De esta forma pasó a formar un bloque indisoluble junto con el taller de prensa. Todas las semanas, sin falta, el taller de radio se encarga de recoger el trabajo 
realizado durante el taller de prensa. Al principio, una recopilación de las noticias internacionales y nacionales recogidas y analizadas bajos los puntos de vista de varios medios. Más adelante empezamos a redactar nuestras propias noticias, tanto noticias de la prensa redactadas con nuestro toque personal, como noticias propias de las actividades protagonizadas por los jóvenes del proyecto educativo. Los primeros "radioboletines" fueron grabados con la ayuda de una mesa de mezclas y un ordenador y colgados en Internet, y consiguieron un efecto multiplicador. El trabajo realizado por los jóvenes, no perece, sino que perdura en la red. Resulta para ellos un estímulo motivador escucharse a través de Internet, analizar sus aciertos y sus fallos, decir a sus amigos y a su familia que escuchen el último programa grabado.

A partir de ahí, el ritmo de trabajo fue aumentando. Programas de deportes, de historia y cultura de la zona, de recetas de cocina, de lecturas recomendadas... Todo es una oportunidad para comunicar a los demás, y todo resulta ser una excusa para aprender. Además, vamos descubriendo cómo el propio lenguaje de la música estimula y motiva a los adolescentes. Empezamos a grabar nuestras propias cuñas publicitarias y nuestras sintonías... Todos desean que llegue la hora de grabar, pero antes, hay que cumplir con otras muchas tareas necesarias para la convivencia en la Casa Escuela. Hay que trabajar en el taller de mantenimiento, o en la huerta, o en la lavandería, o en la cocina, estudiar y hacer los deberes, recoger las habitaciones, y muchas actividades más.

Fruto de otra de las actividades estrella del proyecto educativo, "Dejarse preguntar", surge la sección de entrevistas en radio ESIM. "Dejarse preguntar" es una actividad que realizamos con mucha frecuencia con el fin de aprovechar la presencia de alguna persona concreta en nuestra Casa Escuela o cuando visitamos otros lugares. Se trata de aprovechar al máximo, a través de las preguntas y el diálogo, para aprender sobre una vocación (profesional o de estado) y sobre la vida concreta de una persona. Futbolistas, escritores, historiadores, misioneros, obispos, parados,... han sido entrevistados por los micrófonos de radio ESIM. $\mathrm{Y}$ así, llevados por la propia experiencia, marcada por el entusiasmo, la creatividad, la motivación y el protagonismo de los jóvenes, es como va tomando forma radio ESIM, una radio joven hecha por jóvenes. Hasta el punto de plantearnos en el curso 2010-2011, la construcción de nuestro propio estudio de radio. El proyecto fue acogido con gran entusiasmo por parte de los jóvenes, aún a sabiendas de que todo el trabajo dependía de nosotros mismos. Esto implicaba un sobreesfuerzo en las tareas diarias, pues a la producción creativa de programas, que no se iba a abandonar durante la construcción del estudio, había que sumar la construcción del estudio con nuestras propias manos. Cuatro intensísimos meses de trabajo unidos al trabajo pequeño y diario de varios años atrás, de trabajo constante y organizado, dieron lugar a lo que hoy es el estudio de radio ESIM, y permitieron que pocos meses después de su inauguración, los jóvenes de Camino Juvenil Solidario (los jóvenes de mayor edad de nuestra asociación juvenil) pudieran grabar su primer disco en un estudio que había sido literalmente construido por algunos de ellos.

Este es a grandes rasgos el itinerario de radio ESIM. En él se entremezclan la praxis educativa y la reflexión pedagógica. En él se funden libertad y responsabilidad, educación y comunicación. Cientos de horas grabadas y disponibles en la red, con 
programas de noticias, cuñas, entrevistas, programas especiales... Programas grabados en nuestro querido y sudado estudio, y programas grabados en las calles de nuestras ciudades, con el fin de hacer realidad una escuela abierta al entorno. El Parque del Retiro, la Puerta del Sol, Plaza de España, además de diversos centros educativos y, cómo no, la red, han sido testigos de nuestro deseo de educar(nos) y comunicar.

Para finalizar, podemos concluir que el proyecto de radio ESIM en cuanto a realización práctica, con sus aciertos y errores, pone de manifiesto que el DISEÑO, CONSTRUCCIÓN Y PUESTA EN MARCHA DE UN ESTUDIO DE RADIO en cualquier escuela, constituye un proyecto pedagógico ambicioso, y a la vez realista, capaz de dar sentido tanto a nuestras normativas educativas, como al sentido último de nuestra tarea educativa: la formación integral de hombres y mujeres libres; la construcción de hombres y mujeres responsables con la sociedad en la que les ha tocado vivir; la transformación de edificios muertos (en muchos casos) en escuelas vivas, comprometidas con su entorno y realmente abiertas a él.

Ambicioso, y por ende preñado de creatividad, de espíritu motivador, e innovador, así como de una metodología y un enfoque eminentemente prácticos y funcionales, capaces de provocar en los alumnos verdaderos aprendizajes significativos, impulsados por un modelo de enseñanza-aprendizaje constructivista. Realista, y que hunde sus raíces por tanto en la necesidad de una colaboración interdisciplinar así como en la responsabilidad por parte de todos los agentes que forman la comunidad educativa: equipo educativo, alumnos y familias. Así mismo es inestimable y necesaria la colaboración que puede desarrollar el equipo directivo, la Asociación de
Madres y Padres de Alumnos y las correspondientes corporaciones locales, al tener este proyecto una clara vocación de apertura del centro al entorno. Desde su diseño y construcción (elección de materiales y técnicas, cálculos) hasta su puesta en marcha $\mathrm{y}$ grabación de programas (redacción, locución, elección de temas, software de edición), no sólo entramos de lleno en los contenidos básicos del currículo de varias de las asignaturas (carácter interdisciplinar), sino que estaremos trabajando de una forma práctica la totalidad de las competencias básicas reconocidas por nuestro sistema educativo.

\section{Líneas pedagógicas del proyecto de Radio ESIM}

El diseño, elección de técnicas constructivas y materiales, la construcción y puesta en marcha de un estudio de radio, no se trata exclusivamente de un proyecto tecnológico. Diferentes materiales (pladour, madera, vidrio de seguridad, aluminio, plásticos), con diferentes funciones (resistentes, aislantes, auxiliares), la necesidad del cálculo y diseño de circuitos eléctricos, electrónicos y de sonido, pueden guiar nuestra mirada hacia la herramienta, hacia el QUÉ. Pero no, este proyecto va mucho más allá de su mero carácter instrumental, y hunde sus raíces en la dimensión ética de la profesión docente, teniendo vocación en sí mismo de Proyecto Pedagógico. La enseñanza no es un mero oficio técnico, importando en ella más los FINES (PARA QUÉ) que las herramientas (QUÉ). (Fullan, 1997). Como proyecto con vocación de Proyecto Pedagógico, se tiene en cuenta los tres polos sobre los que se sustenta todo modelo pedagógico, el Axiológico (¿Desde dónde?, ¿Para qué?), el de Apuntalamiento Psicológico (¿A 
quién?) y el Praxeológico (¿Cómo?, ¿Con qué?). (Meirieu, 2001). Por tanto, este proyecto se sustenta en unos principios y fines mucho más amplios como el diálogo, la conciencia crítica, el trabajo y la colaboración, la motivación y la solidaridad, además de tener una clara vocación de apertura a la sociedad, superando las fronteras del contexto escolar. Se trata pues de un aprendizaje instrumental a la vez que comunicativo y dialógico, superando así la dialéctica y contraposición entre ambos tipos de aprendizaje que desde posturas tanto progresistas como tradicionales se ha venido manteniendo en los últimos años. (Bloom, 1989) citado en (Flecha, 2008)

Como líneas pedagógicas, se basa en las siguientes:

- Una Pedagogía del Diálogo y de la Comunicación. Aprendizaje dialógico: Todo lo realizado en el proyecto está basado en el diálogo, la escucha y la acción comunicativa. Un aprendizaje dialógico desde la propia toma de decisiones a la puesta en marcha final del proyecto y el trabajo propiamente comunicativo e interdisciplinar.

- Una Pedagogía de la Conciencia Crítica: que ayude al joven a descifrar el mundo en el que vive, y la necesidad de su compromiso con la sociedad. En una pared de nuestra escuela está escrito con letras grandes: "I care"'. Es la divisa intraducible de los mejores jóvenes americanos. "Me importa, me preocupa". (Milani, 1995)

- Una Pedagogía del Trabajo y la Colaboración: A lo largo de la historia de la educación, muchas han sido las teorías pedagógicas que han incorporado el trabajo en el proceso de Enseñanza-
Aprendizaje. El proyecto de construcción de un estudio de radio, con la colaboración de los propios alumnos, busca tener en cuenta el valor educativo inherente al trabajo, así como que los alumnos hagan suyo, protagonicen el proyecto. En este sentido resulta clarificadora la siguiente reflexión:

«Las mismas ideas que es capaz de producir el entendimiento, no adquieren categoría hasta que han pasado por las manos. Las ideas que elabora la mente y la boca, se las lleva el viento y carecen de consistencia hasta que una mano las ha escrito, o esculpido, o realizado de cualquier forma o manera» (Rovirosa, 2009) citado en (Rodríguez Peralta, 2011)

- Una Pedagogía de la Motivación: Como han puesto de manifiesto varios trabajos recientes, cuando los psicólogos han investigado de qué depende que los escolares se esfuercen y se interesen por aprender, han comprobado que el interés y el esfuerzo varían según sea la respuesta a cuatro preguntas que las personas nos planteamos antes y a lo largo de una actividad. Estas preguntas son:

¿Qué tengo que conseguir?: Se refiere al Objetivo. ¿Qué satisfacción voy a obtener?: Se refiere a las Metas.

¿Qué efectos negativos tendrá?: Se refiere al Coste. ¿Qué hacer para conseguirlo?: Se refiere a los Medios, y afecta a las Expectativas.

(Alonso Tapia, 2005)

Además de tener en cuenta estos elementos, es más que evidente que el proyecto de estudio de radio, en 


\section{II: Radio y educación. Perspectivas y contextos}

sí mismo, tiene, por cercanía a las características del desarrollo psicológico y evolutivo de los adolescentes, elementos lo suficientemente motivadores como para captar la atención y el compromiso de los mismos.

- Una Pedagogía Solidaria: las prácticas educativas igualitarias sólo pueden fundamentarse en concepciones solidarias. La teoría de la acción comunicativa de Habermas, la perspectiva emancipadora de Freire, y muchas otras teorías y prácticas educativas llevan a afirmar rotundamente que democracia, igualdad, paz o libertad son más deseables que dictadura, desigualdad, guerra o esclavitud, y que la educación tiene que trabajar a favor de las primeras y en contra de las segundas (Flecha, 2008).

- En definitiva, una pedagogía para la vida, que permita a nuestros jóvenes decir, como aquellos alumnos de Barbiana:

«He aprendido que el problema de los demás es igual al mío. Salir de él todos juntos es la política. Salir solo, la avaricia» (Alumnos de Barbiana, 2009)
Algunas imágenes del proyecto (1)

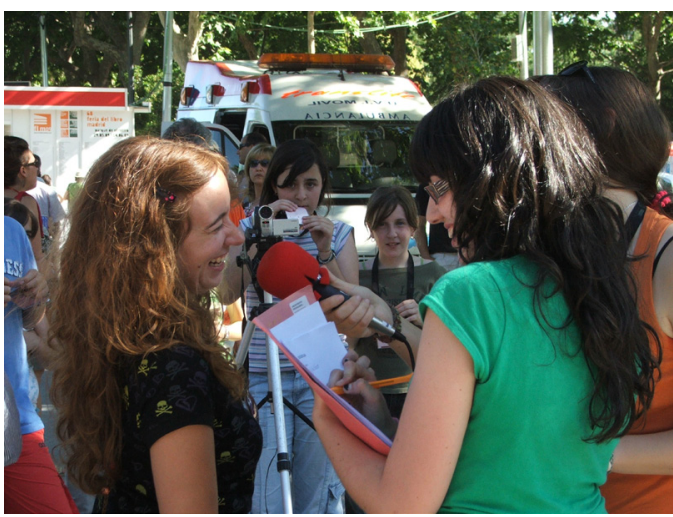

Entrevistas en el Día del Libro en el Parque del Retiro de Madrid

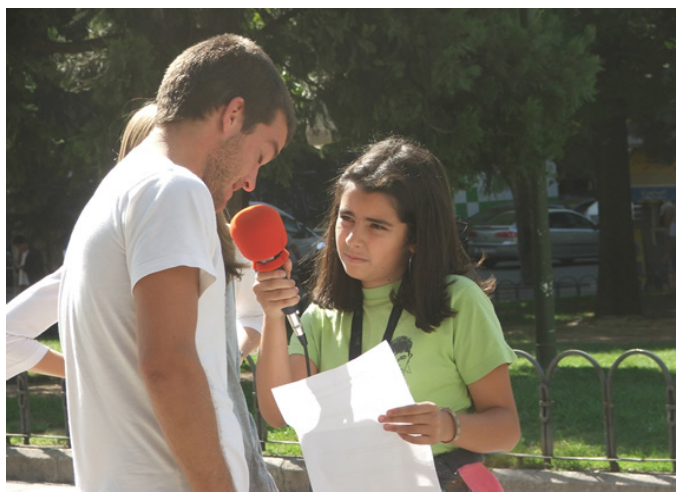

Entrevistas sobre el paro y la situación económica En Plaza de España, Madrid 


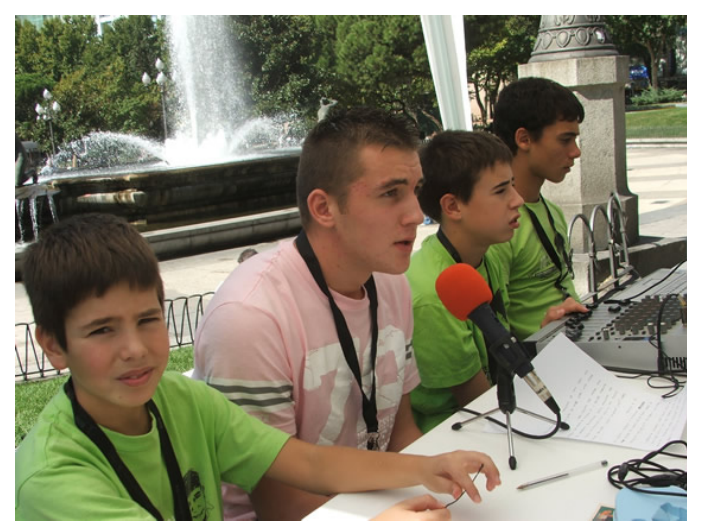

Programa especial en Plaza de España, Madrid

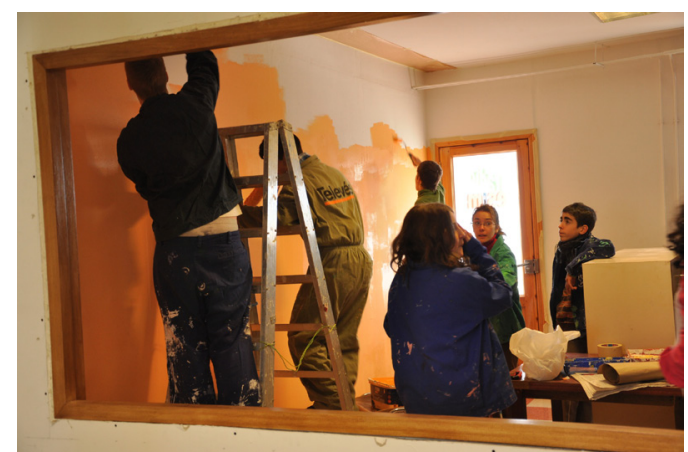

Uno de los momentos durante la construcción del estudio

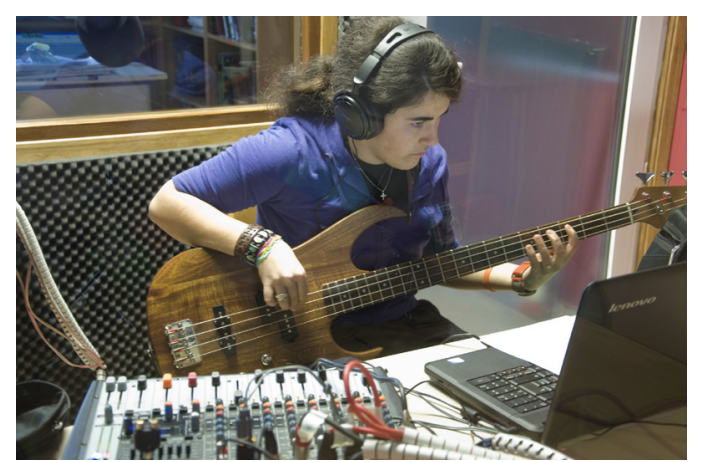

Grabación de sintonías para radio ESIM en el nuevo estudio

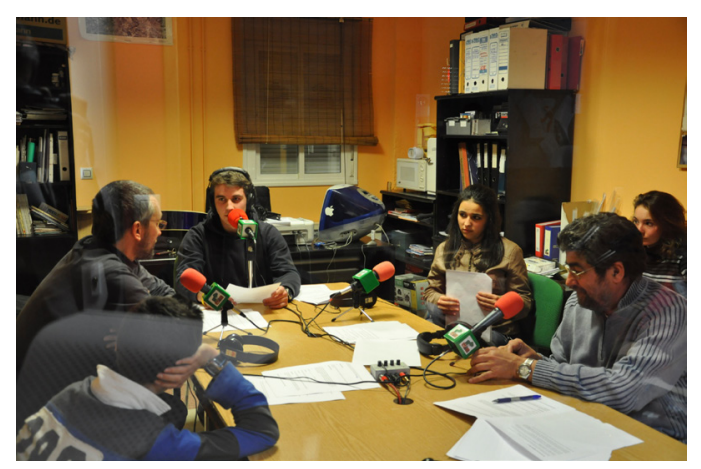

Programa inaugural del estudio de radio ESIM, al que acuden familiares, vecinos del pueblo y profesores del IES Alto Jarama de Torrelaguna. 


\section{II: Radio y educación. Perspectivas y contextos}

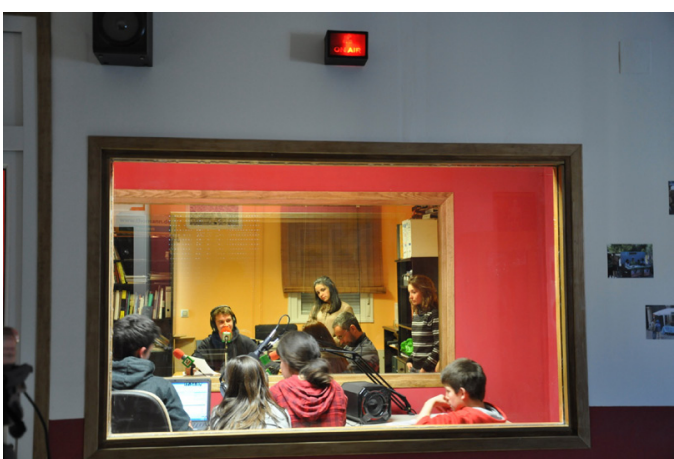

Los jóvenes técnicos de radio ESIM contemplan $\mathrm{y}$ siguen atentamente el directo inaugural $\mathrm{y}$ las entrevistas realizadas por los también jóvenes reporteros de radio ESIM.

Más información y acceso a programas grabados por los jóvenes de radio ESIM en:

www.solidaridad.net/esim/audio/radio_ESIM/radio. php

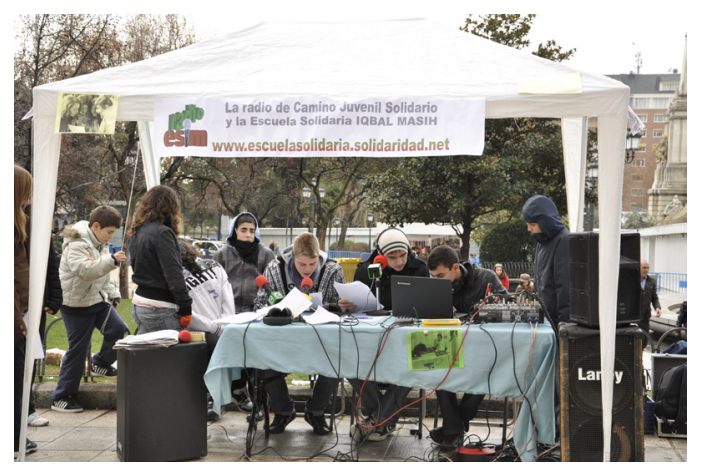

\section{Notas}

(1) Pueden conseguirse las que se necesiten a través del autor

\section{Referencias}

Alonso Tapia, J. (2005). Motivar en la escuela, motivar en la familia. Madrid: Ediciones Morata.

Alumnos de la Escuela de Barbiana. (2009). Carta a una maestra. Madrid: Voz de los Sin Voz.

Amar, V. (2010). "La educación en medios digitales de comunicación", en Pixel Bit. Revista de Medios y Educación, 36; 115-124.

Bronfenbrenner, U. (1987). La ecología del desarrollo humano. Barcelona: Paidós.

Cervera, J. (1977). Otra escuela. Cine - Radio - TV Prensa. Madrid: Ediciones S.M.

Dieuzeide, H. (1984). "Comunicación y Educación”, en UNESCO: la Educación en materia de Comunicación. UNESCO.

Flecha, R. (2008). Compartiendo Palabras. El aprendizaje de las personas adultas a través del diálogo. Barcelona: Paidós.

Freire, P. (1990). La naturaleza política de la educación. Cultura, poder y liberación. Barcelona: Paidós y Ministerio de Educación y Ciencia.

Fullan, M., y Hargreaves, A. (1997). ¿Hay algo por lo que merezca la pena luchar en la escuela? Trabajar unidos mara mejorar. Sevilla: MCEP; 41-43.

Medina Rodríguez, J. J. (2010). "Una investigación evaluativa: La Casa Escuela Iqbal Masih. Una iniciativa de los padres en la educación no formal". Tesis Doctoral no publicada, Universidad de Granada: Granada. 
Meirieu, P. (2001). La opción de educar. Ética y pedagogía. Barcelona: Octaedro; 113-130.

Milani, L. (1995). Dar la palabra a los pobres. Cartas de Lorenzo Milani. Madrid: Acción Cultural Cristiana. Moles, A. A. (1969).

Rodríguez Peralta, M. I. (2007). "El método de Encuesta como estrategia de aprendizaje. Una experiencia concreta en la formación inicial del profesorado". Tesis Doctoral no publicada, Universidad de Granada, Granada.

Rovirosa, G. (2009). Obras Completas. Tomo IV. Madrid: Ediciones HOAC.

Trilla Bernet, J. (2003). La educación fuera de la Escuela. Ámbitos no formales y educación social. Barcelona: Ariel. 